\title{
Star-Planet Interaction: The Curious Case of the Planet Spoon-feeding Its Host Star (and Other Amenities)
}

\author{
Ignazio Pillitteri ${ }^{1,2}$ and S. J. Wolk ${ }^{1}$ and A. Maggio ${ }^{2}$ and T. Matsakos ${ }^{3}$ \\ ${ }^{1}$ SAO-Harvard CfA, 60 Garden St. Cambridge 02138 MA, USA \\ email: ipillitt@cfa.harvard.edu \\ ${ }^{2}$ INAF-Osservatorio Astronomico di Palermo, Piazza del Parlamento 1, 90134 Palermo, IT \\ ${ }^{3}$ Dept. Astronomy \& Astrophysics, University of Chicago, 5640 S. Ellis Ave, Chicago, IL \\ 60637, USA
}

\begin{abstract}
We report two cases of Star-Planet Interaction (SPI) in two systems with hot Jupiters: HD 189733 and HD 17156. We used HST-COS to study the FUV variability of HD 189733 after the planetary eclipse. With the support of MHD simulations, we evince that material is likely evaporating from the planet and accreting onto the parent star. This produces a hot spot on the stellar surface, co-moving with the planetary motion and responsible of the X-ray and FUV variability at peculiar planetary phases. In HD 17156, which hosts a hot Jupiter in an eccentric orbit, we observed an enhancement of the X-ray activity at the passage of its planet at the periastron. The origin can be due to magnetic reconnection between the planetary and stellar magnetic fields, or due to material tidally stripped from the planet and accreting onto the star.
\end{abstract}

Keywords. stars: activity, stars: coronae, stars: solar-type, (stars:) planetary systems, stars: individual (HD 189733, HD 17156)

\section{Introduction}

Hot Jupiters are close-in exoplanets inflated by the strong radiation from their host stars and lose their atmospheres. There is evidence that hot Jupiters can interact through magnetic fields and gravity with their parent stars, influencing the stellar activity at a detectable level. These phenomena constitute cases of Star-Planet Interaction (SPI).

\section{HD 189733}

In FUV observations of HD 189733 with HST COS during and after a planetary eclipse we found two episodes of strong variability of the line fluxes of ions of $\mathrm{Si}, \mathrm{C}$, and $\mathrm{N}$ that have not been observed in previous observations at planetary transits. The variability at the post planetary eclipse is analogous to the X-ray variability seen with XMM-Newton (Pillitteri et al. $2010,2011,2014$ ) and points to stellar activity phased with the planetary motion. We interpret this activity as the signature of material detaching from the planet and falling onto the star (Fig. 1). This accretion creates a hot spot moving in phase with the planet and observed when it is emerging at the stellar limb (Pillitteri et al., 2015; Matsakos et al., 2015).

\section{HD 17156}

We have also studied the case of HD 17156, a system with a hot Jupiter in a very eccentric orbit. HD17156 is a G0 star with a hot Jupiter in a 21 days period orbit (Barbieri et al. 2007). The eccentricity of the orbit is 0.68 , and the planet reaches a minimum separation of $\sim 7 R_{*}$. We observed HD 17156 in September 2014 with XMM-Newton and HARPS-N when the planet was at 



Figure 1. Particle density contours $\left(\right.$ units $^{-3} \mathrm{~cm}^{-3}$ ) of an MHD simulation that models star-planet interactions (Matsakos et al., 2015) between a hot Jupiter and its host (polar view). The star rotates counterclockwise, and the planet orbits the star along the same direction. The two "+" symbols shown on the left panel indicate the location of the star (red disk) and the planet (green disk). A close up of the impact region is depicted in the right panel, where the motion of the accreting plasma is marked with arrows. The shocked plasma is funnelled by the magnetized stellar wind in an almost radial trajectory close to the star (A), it forms a "knee" structure that consists of hot and dense plasma (B), and then accretes in a spot ahead of the orbital phase (C).
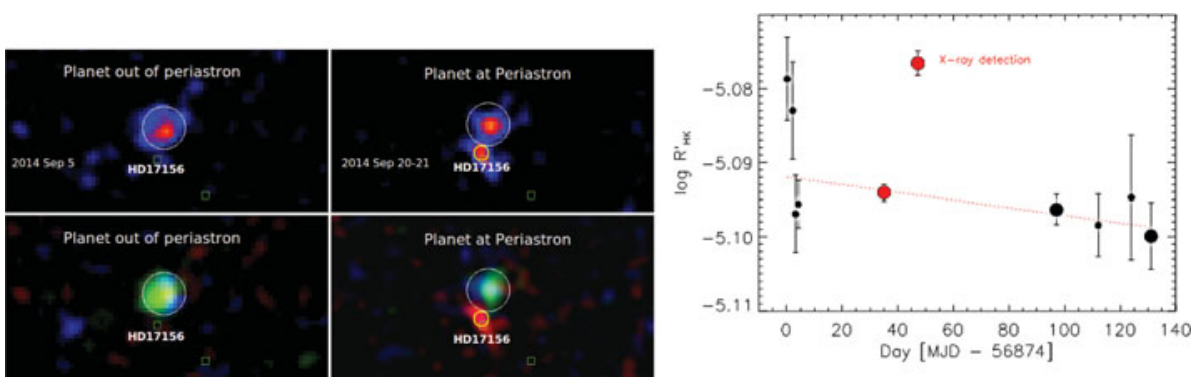

Figure 2. Left: XMM-EPIC count images (top panels) and $r g b$ composite images (bottom panels) of HD 17156 during two XMM-Newton visits. Circles show the position of the detection of HD 17156, boxes are Simbad objects. An unknown X-ray source is also detected at $~ 10$ " from our target. Right: $\log R_{H K}^{\prime}$ vs. MJD. Red solid symbols are the observations coordinated with $X M M$-Newton visits. Symbol sizes are proportional to the exposure times of the spectra.

the periastron and when it was farther out. We found an increase of coronal and chromospheric activity that suggests a systematic variability during periastron passages (Fig. 2). The cause might be either due a magnetic reconnection (i.e., flaring activity) when the planet was at its minimum separation, or due to material stripped from the planet and falling onto the star. The excess of X-rays has a soft spectrum and this favors the tidal stripping hypothesis.

\section{References}

Barbieri, M., Alonso, R., Desidera, S., et al. 2009, A\&A, 503, 601

Matsakos, T., Uribe, A., \& Königl, A. 2015, A\&A 578A, 6

Pillitteri, I., Maggio, A., Micela, G., et al. 2015, ApJ 805, 52

Pillitteri, I., Wolk, S. J., Lopez-Santiago, J., et al. 2014, ApJ, 785, 145

Pillitteri, I., Günther, H. M., Wolk, S. J., Kashyap, V. L., \& Cohen, O. 2011, ApJL, 741, L18

Pillitteri, I., Wolk, S. J., Cohen, O., et al. 2010, ApJ, 722, 1216 